Note on the Bitangents of a Plane Curve

Author(s): A. P. Thompson

Source: The Mathematical Gazette, Vol. 2, No. 40 (Jul., 1903), pp. 307-308

Published by: Mathematical Association

Stable URL: http://www.jstor.org/stable/3605127

Accessed: 29-10-2015 10:54 UTC

Your use of the JSTOR archive indicates your acceptance of the Terms \& Conditions of Use, available at http://www.jstor.org/page/ info/about/policies/terms.jsp

JSTOR is a not-for-profit service that helps scholars, researchers, and students discover, use, and build upon a wide range of content in a trusted digital archive. We use information technology and tools to increase productivity and facilitate new forms of scholarship. For more information about JSTOR, please contact support@jstor.org. 


\section{NOTE ON THE BITANGENTS OF A PLANE CURVE.}

The equation of the curve of order 14, on which lie the points of contact of the 28 bitangents of a quartic curve, was first given by Hesse (Crelle's Journal, Vols. 36, 40, 41). A very concise form of the equation in the Clebsch-Aronhold symbols was obtained by Dersch (Math. Ann., Bd. 7). The method adopted in his Memoir was practically that given by Salmon (Phil. Mag., Oct. 1858), and followed by Cayley (Phil. Trans., 1859).

In 1886 appeared Maisano's paper (Math. Ann., Bd. 29) containing the equation to the curve on which lie the contacts of the bitangents of the general quintic curve.

In the present Note, attention is drawn to a certain covariant curve belonging to the plane curve considered.

Let $f(x, y, z)=0$ be the equation in homogeneous point-coordinates to a curve of order $n$, which we shall assume non-singular.

A point $\left(x^{\prime}, y^{\prime}, z^{\prime}\right)$ has, with respect to $f$, a set of polar curves

$$
\begin{array}{r}
\Omega f=0, \\
\Omega^{2} f=0, \\
\cdots . . . . . \\
\Omega^{r} f=0, \\
\ldots . . . . .
\end{array}
$$

where

$$
\Omega \equiv x^{\prime} \frac{\partial}{\partial x}+y^{\prime} \frac{\partial}{\partial y}+z^{\prime} \frac{\partial}{\partial z}
$$

called the first, second, ... $r$ th, ... polar curves of $x^{\prime} y^{\prime} z^{\prime}$.

The $(n-1)$ th polar curve of a point is a straight line ; and every line has $(n-1)^{2}$ poles.

The covariant curve under discussion is the locus of the poles of the tangent lines to the given curve $f$.

One of the $(n-1)^{2}$ poles of the tangent to $f$ at the point $P$ is evidently the point $P$ itself, so that the ground curve $f$ is part of the locus. The remainder of the locus constitutes a covariant curve $\phi=0$ of order $n^{2}(n-2)$, cutting $f$ in its bi-contact points, and having inflexions with the same tangent line at the inflexions of $f$-thus verifying the number of bi-contacts of $f$ from the identity

$$
2 \cdot \frac{1}{2} n(n-2)\left(n^{2}-9\right)+3 \cdot 3 n(n-2)=n \cdot n^{2}(n-2) \text {. }
$$

We proceed to prove these theorems.

The equation $\phi=0$ can be found by writing $\frac{\partial f}{\partial x}, \frac{\partial f}{\partial y}, \frac{\partial f}{\partial z}$ for the linecoordinates in the tangential equation of $f$, and dividing out the factor $f$.

The tangential equation of the non-singular curve $f$ is of order $n(n-1)$ in the line-coordinates. Substituting $\frac{\partial f}{\partial x}, \ldots$ which are of order $(n-1)$ in the point-coordinates, and dividing out $f$, the remaining locus is of order

$$
n(n-1)^{2}-n=n^{2}(n-2) \text {. }
$$

A bitangent to $f$, having contact at $P$ and $Q$, has $P, Q$ for two of its $(n-1)^{2}$ poles. Hence $P$, corresponding to the tangent at $Q$, lies on $\phi$. Similarly for all the bi-contacts.

Let $O$ be a point of inflexion on $f$. Referred to the tangent and normal at $O$ as $x, y$ axes, the equation of $f$ in the neighbourhood of $O$ takes the form

$$
y=\alpha x^{3}+\beta x^{4}+\text { higher powers. }
$$


The polar line of $x^{\prime}, y^{\prime}$ is

$$
x\left\{3 \alpha x^{\prime 2}+4 \beta x^{\prime 3}+\ldots\right\}-y+\left\{-(n-1) y^{\prime}+\alpha(n-3) x^{\prime 3}+\ldots\right\}=0 .
$$

The tangent-line at $x^{\prime \prime}, y^{\prime \prime}$, a point on $f$ very near $O$, is

$$
x\left\{3 \alpha x^{\prime \prime 2}+4 \beta x^{\prime \prime 3}+\ldots\right\}-y+\left\{-2 \alpha x^{\prime \prime}+\ldots\right\} .
$$

Now since an inflexion may be regarded as the limiting point of coincidence of the two contacts of a bitangent, the curve $\phi$ must pass through $O$; and the polar line of a point $x^{\prime}, y^{\prime}$ on $\phi$ very near $O$ will coincide with the tangent at $x^{\prime \prime}, y^{\prime \prime}$ on $f$ near $O$, to the first order of smallness, if

$$
x^{\prime 2}=x^{\prime \prime 2} \text { and }(n-1) y^{\prime}-(n-3) \alpha x^{\prime 3}=2 \alpha x^{\prime \prime 3} .
$$

Rejecting the solution $x^{\prime}=x^{\prime \prime}$ corresponding to the point of contact, the pole of the line near its contact is given by

$$
x^{\prime}=-x^{\prime \prime}, y^{\prime}=-\frac{n-5}{n-1} \alpha x^{\prime \prime 3},
$$

to the first order of small quantities. Hence the curve $\phi$ in the neighbourhood of $O$ approximates to the form of the curve

$$
y=\frac{n-5}{n-1} a x^{3},
$$

in other words has an inflexion at $O$ with the same tangent there as $f$.

It may be verified without difficulty that the equation of $\phi$ for the cubic

is of the form

$$
f \equiv x^{3}+y^{3}+z^{3}+6 m x y z
$$

$$
\sum x^{9}+6 m x y z \sum x^{6}+\alpha . \Sigma\left(y^{3} z^{6}+y^{6} z^{3}\right)+\beta \cdot x^{2} y^{2} z^{2} \cdot \Sigma x^{3}+\gamma \cdot x^{3} y^{3} z^{3},
$$

where $\alpha, \beta, \gamma$ are numerical, a curve of order 9 having inflexions at all those of the cubic.

It may be remarked that if $C=0$ be the equation to the curve found by Hesse, Salmon, and others, the above curve $\phi$ can be written

$$
\phi \equiv A f+C H^{3},
$$

where $A$ is a quantic of order $n^{2}(n-2)-n$.

We can connect $\phi$ with the Steinerian of $f$ as follows :

The Steinerian is a curve of order $3(n-2)^{2}$, class $3(n-1)(n-2)$, which is the locus of a point whose first polar for $f$ has a node-and is also the envelope of line-polars of points of the Hessian.

It is known that the Steinerian touches all the inflexional tangents to $f$....(2), and by $(1)$, if the point $(x, y, z)$ lies on the Hessian, the line whose coordinates are

touches the Steinerian.

$$
l: m: n=\frac{\partial f}{\partial x}: \frac{\partial f}{\partial y}: \frac{\partial f}{\partial z}
$$

Suppose now $(x, y, z)$ lies also on $\phi$. Then the line

$$
l: m: n=\frac{\partial f}{\partial x}: \frac{\partial f}{\partial y}: \frac{\partial f}{\partial z}
$$

touches $f$. Hence to every intersection of $\phi$ and $H$ corresponds a common tangent to $f$ and the Steinerian $S$.

Now the inflexional tangents to $f$ are common tangents to $f$ and $S$ by (2), and since such a tangent to $f$ is a stationary tangent, each must count twice as a common tangent to $f$ and $S$. Thus since $\phi, H$ have $3 n^{2}(n-2)^{2}$ common points, $f$ and $S$ have $3 n^{2}(n-2)^{2}+3 n(n-2)$ common tangents. This number $=3 n(n-2)(n-1)^{2}$, and $f$ is of class $n(n-1)$. Hence the Steinerian must be of class $3(n-1)(n-2)$, verifying a known result.

A. P. Thompson. 